\section{DE ABSTRACTO A CONCRETO}

Pedro Alonso

Profesor, Pontificia Universidad Católica de Chile

Hugo Palmarola

Investigador invitado, Indiana University

Durante la segunda mitad del s. Xx se desarrollaron notables experiencias de prefabricación en grandes paneles de hormigón, aplicadas en la construcción masiva de viviendas. La revisión de estas experiencias puede dar claves para renovar el potencial de estos sistemas.

Palabras clave: Prefabricación, prefabricados de hormigón, vivienda colectiva, sistema constructivo.

During the second half of the $20^{\text {th }}$ century, the use of precast concrete panels to build large housing projects became a significant prefabrication practice. Revisiting these systems can provide keys to understand the current potential of prefabrication.

Keywords: Prefabrication, concrete prefabs, collective housing, building system.

i bien marginales al canon historiográfico de la arquitectura moderna, el desarrollo de sistemas constructivos prefabricados a partir de grandes paneles de hormigón armado fue central en los debates sobre modernización e industrialización de la construcción. Estas discusiones excedieron al ámbito meramente técnico o práctico para transformarse en arena de especulación política, estética e ideológica, tanto en arquitectura como en el campo del arte. Esto queda de manifiesto en la proliferación de sistemas de edificación que tuvo lugar en países tan distintos -y distantes- como Francia, la entonces Unión Soviética, Suiza, República Democrática Alemana, Reino Unido, Yugoeslavia, Canadá, Estados Unidos, Dinamarca, Holanda, Japón, Cuba y Chile, entre otros. En la Unión Soviética (tal vez el ejemplo más paradigmático), este desarrollo estuvo marcado por el célebre discurso de Nikita Khrushchev ante la Conferencia Nacional de Constructores, Arquitectos y Trabajadores en 1954 y cuyo objetivo era la "desestalinización" del país y la eliminación de los excesos ornamentales propios del realismo socialista, apelando a una ética y a una estética constructiva resumida en su valoración de la expresión de las líneas de junta entre paneles -o que denominó como "línea de la honestidad"-. Estos sistemas y sus respectivas tipologías edificatorias serían conocidos consecuentemente como Khrushchukas.

Denominadas genéricamente en la URss a través de siglas $-\mathrm{K}_{7}$; G-3i; G-5; II-32; II-35; II-49; II-57; 1MG-300; 1-335; 1-510; 1-605; II-57 o 1-464- las Khrushchvkas interesaron a Georges Maciunas, fundador de Fluxus, quien publicó en 1965 el panfleto titulado Communists must give revolutionary leadership in culture. Ahí presentaba la prefabricación soviética de grandes paneles como la arquitectura que lograba una mayor eficiencia, dado su completo desinterés por tradiciones artísticas nacionales y estilísticas, y la producción en cambio de soluciones a problemas de diseño utilizando los últimos avances tecnológicos (Flynt y Maciunas, 1965). Las Khrushchvkas ejemplificaban, para Maciunas, los tres principios esenciales para lograr un liderazgo revolucionario en cultura: incremento de la productividad, promoción de la igualdad y solidaridad entre trabajadores, y satisfacción del deseo de los trabajadores de confrontar la realidad. En este contexto de especulación artística y debate político es de interés que la diversificación y riqueza tecnológica y tipológica de estas series aún parece ser un campo relativamente inexplorado de investigación ${ }^{1}$, más aún si se considera que a nivel mundial estos sistemas se emplearon para construir alrededor de 190.000.000 de departamentos.
A través de su modelación tridimensional en el software Rhinoceros y la fabricación digital de modelos en escala 1: 75 -por medio de una impresora de polvo $\mathrm{Z}_{310}$ plus- se propuso a estudiantes de séptimo semestre la reconstrucción técnica, tipológica, histórica y conceptual de este tipo de sistemas. El Taller de Investigación "De abstracto a concreto" desarrollado en la Escuela de Arquitectura de la Universidad Católica en 2011 y 2012, permitió ampliar la reflexión crítica sobre la historia de la prefabricación a partir de una metodología que utilizó el modelo digital para estudiar las relaciones cambiantes entre arquitectura y tecnología durante el siglo xx. ${ }^{2}$

"De abstracto a concreto" mostró que estos sistemas estaban lejos de la monotonía y la rigidez tipológica con que usualmente se les caracteriza. Por el contrario, la gran cantidad de sistemas creados, su evolución y adaptación a contextos sociales, técnicos, geográficos y culturales diversos es evidencia de una voluntad de variación que buscaba alternativas cada vez más eficientes -desde un punto de vista económico y estratégico- en la solución de los grandes problemas de la provisión de vivienda económica y colectiva. Específicamente, el taller investigó los sistemas Camus y Coignet (Francia); K7, 1-335, 1-464, E-101 y 1-510 (URss); Gran Panel Soviético (Cuba); KPD у VEP (Chile); Larsen $\mathcal{E}$ Nielsen (Dinamarca) y Brecast (Inglaterra). Se dejaron para una segunda etapa Descon-Concordia (EE.UU./ Canadá); Gran Panel Iv y Gran Panel 70 (Cuba); Wohnungsbauserie 70 (RDA); Jugomont 61 (Yugoeslavia); vAM (Holanda); Igeco (Suiza); Taisei (Japón) y de manera especial, el Dormitorio Saint Andrews de James Stirling (Escocia).

La aparente escasez de investigaciones sobre estos esfuerzos de sistematización industrial del hormigón -o al menos la ausencia de estudios comparados que vinculen aspectos técnicos y tipológicos con debates teóricos e históricos- sumado a la imposibilidad de acceder a fuentes primarias muchas veces en ruso, francés, holandés, japonés o alemán, fueron considerados restricciones propias del proceso de reconstrucción de los sistemas. Estas dificultades fueron entendidas como un desafío al objetivo propuesto a los estudiantes: la reconstrucción de sistemas y edificios más bien desconocidos, tanto por su estatus ambiguo en la historiografía de la arquitectura moderna, como por tratarse de obras cuyas lógicas proyectuales y

1 Sí es posible encontrar trabajos más bien recopilatorios, mayoritariamente de carácter referencial o técnico.

2 Los resultados de este trabajo fueron expuestos en la Galería de arte González \& González en Santiago en enero de 2012 y posteriormente en la muestra São Paulo Calling, proyecto curatorial dirigido por Stefano Boeri en colaboración con la Secretaria Municipal de Habitaçao de la Prefeitura do Municipio de São Paulo, en el Centro Cultural São Paulo entre los meses de enero y junio de 2012. 
de producción -al reemplazar al arquitecto individual por la idea de diseño colectivo en manos de trabajadores anónimos- se alejaron de prácticas arquitectónicas convencionales en términos de su difusión y publicación. De este modo, a pesar de su aparente claridad y racionalidad constructiva, la configuración interna y especificidad técnica de estos sistemas aparecía como un misterio.

En el contexto de estas restricciones, el taller reflexionó en torno a la noción de indicio, y recurrió a toda información que permitiera avanzar en la comprensión y reconstrucción de estos sistemas, tanto si se tratase de fuentes primarias (en los casos chilenos) como de datos obtenidos de libros y revistas de época, además de la pesquisa de datos en Internet vía e-mail, GoogleTranslator y Street View y la identificación y contacto de personas a través de redes como Facebook, Skype, Linkedln que permitió entrevistar a arquitectos o ingenieros involucrados originalmente en el desarrollo de estos sistemas ${ }^{3}$. Así entendido, el cruce de información se hizo fundamental en el gradual esclarecimiento de sistemas constructivos a partir de datos fragmentarios o dispersos que, en algunos casos, no excedían información relativa a un solo panel, un par de diagramas o un puñado de fotografías.

Esto se tradujo en estrategias de investigación y análisis basadas en una analogía biológica, propia de la recepción que las ideas del científico francés Georges Cuvier tuvieron en algunos discursos de la arquitectura moderna. En efecto, Cuvier propuso que, a partir del estudio cuidadoso de un solo hueso, uno "debería ser capaz de reconstruir el animal competo al cual este pertenecía” (Cuvier, 1812). Esta aproximación permitió no solo aventurar la reconstrucción del edificio a partir de un solo panel, sino además situar los sistemas, su desarrollo y “evolución' en series, como si se tratase de un problema paleontológico (entendido como la capacidad de reconstruir una especie extinta a partir de algunos restos fósiles). La relación entre Cuvier y este acercamiento no es casual, pues fue él quien en el siglo xviı estableció las bases de la paleontología a partir de su trabajo en anatomía comparada; el estudio de fósiles permitiría determinar la evolución de los organismos y su interacción con el medioambiente. Tras la "extinción" (a fines de los años setenta) de los sistemas prefabricados basados en grandes paneles de hormigón armado, el taller entonces consideró al panel como un resto fósil, y la paleontología como una "ciencia histórica" en su intento por explicar causas en vez de realizar experimentos para observar efectos.

Esta idea -tomada de Cuvier- de una ciencia histórica basada en el estudio de fragmentos, no sería ajena a las investigaciones posteriores de Charles Darwin, quien sostendría que: “...el registro

\section{Esta aproximación permitió no solo aventurar}

\section{la reconstrucción del edificio a partir de un}

solo panel, sino además situar los sistemas, su

desarrollo y “evolución’ en series, como si se tratase de un problema paleontológico entendido

como la capacidad de reconstruir una especie

extinta a partir de algunos restos fósiles.

geológico en tanto historia del mundo está conservado de manera imperfecta y escrito en un dialecto cambiante; esta es una historia además de la cual solo poseemos el último volumen... Y de este volumen, solo un breve capítulo ha sido preservado; y de este capítulo, de cada página, solo algunas pocas líneas aquí y allá son aun accesibles” (Darwin, 1859). Esto explica, según Gillian Beer, que Darwin “aprendió a mirar, a interpretar y, sobre todo, a escribir y usar la escritura como parte de un proceso creativo. A través de la interacción entre experiencia sensible, registro, recolección, revisión y reevaluación, fue adquiriendo la confianza para pensar teóricamente, para aventurar hipótesis y someterlas a escrutinio cuidadoso, sin caer en un empirismo banal. Darwin no pretendía lamentar la ausencia de evidencia, sino reconocer la condición irrecuperable de lo que se ha perdido (Beer, 2008).

Así, la reconstrucción de sistemas constructivos extintos no consistió en abusar de una metáfora biológica, sino más bien de ejercitar un proceso intelectual dotado de profundidad histórica y teórica, donde a partir de la recolección, registro, observación y representación del material disponible y del uso de la escritura como parte de un proceso creativo, se formularan hipótesis de trabajo basadas en "suposiciones informadas". En suma, se trató de proponer para cada sistema una tesis proyectual adecuada.

Tal como una aproximación de este tipo le permitió a Darwin desafiar la idea convencional de variación morfológica a partir de formas arquetípicas o ideales (Beer, 2008), estas hipótesis debían resolver técnicamente el ensamblaje de componentes (muchos de ellos desconocidos) a partir de vestigios y del estudio de las pequeñas diferencias y desviaciones entre sistemas. Se trató de observar en los paneles fósiles las diferencias graduales propias de 


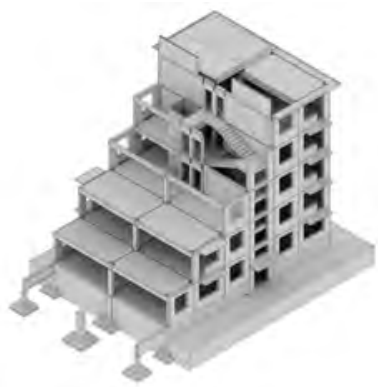

Axonométrica escalonada sistema 1-335, URSS, años cincuenta.

Modelo de Pablo Muñoz.

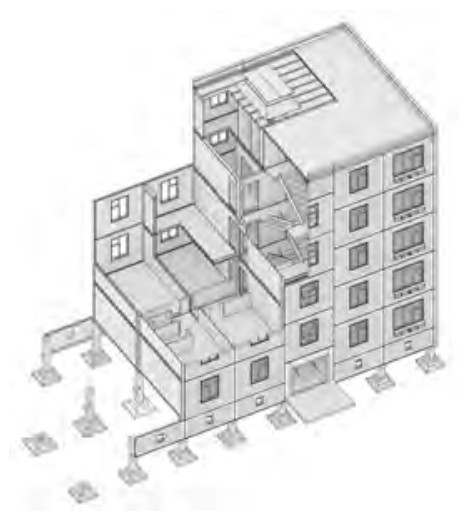

Axonométrica escalonada sistema K7,

URSS, años cincuenta.

Modelo de Ismael Cárdenas.

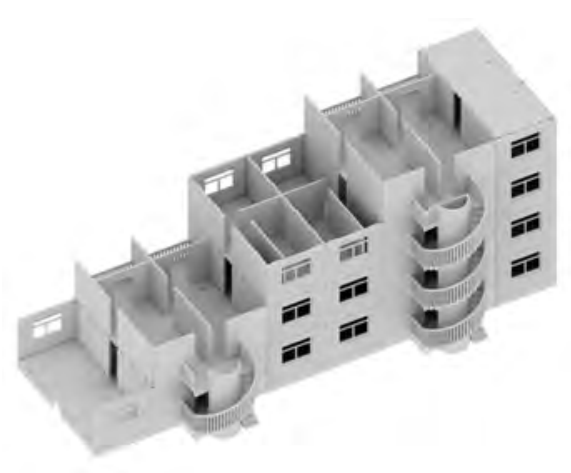

Axonométrica escalonada sistema Brecast, Inglaterra, años sesenta y setenta.

Modelo de Constanza Krauss.

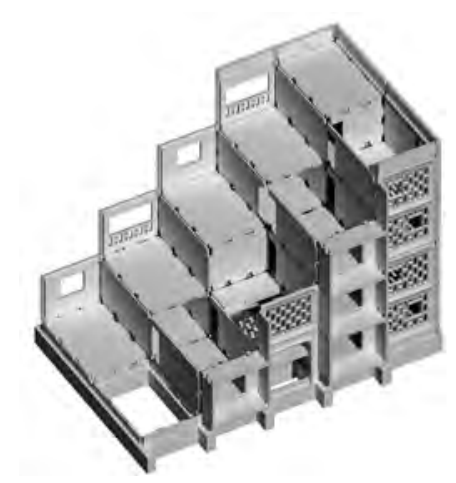

Axonométrica escalonada sistema KPD,

Chile, años setenta.

Modelo de Carolina Rocco.

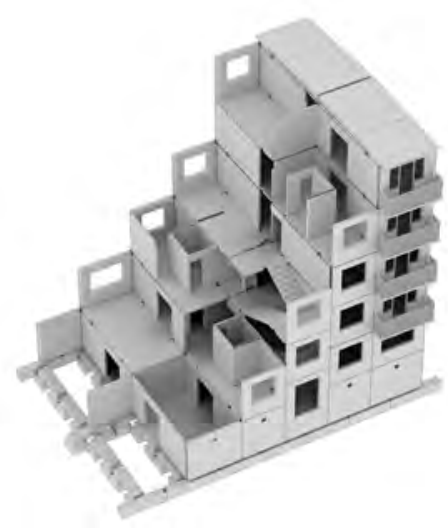

Axonométrica escalonada sistema 1-464, URSS, años cincuenta.

Modelo de Carlos Rojas.

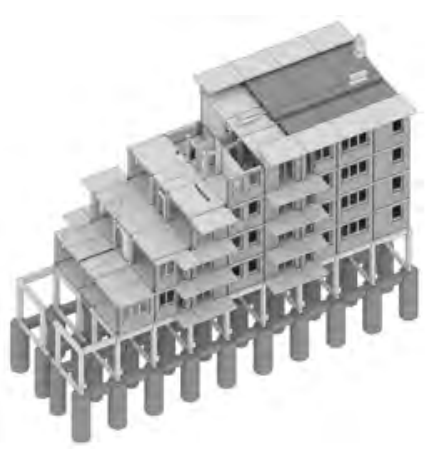

Axonométrica escalonada sistema Camus, Francia, años cincuenta y sesenta. Modelo de David Icekson.

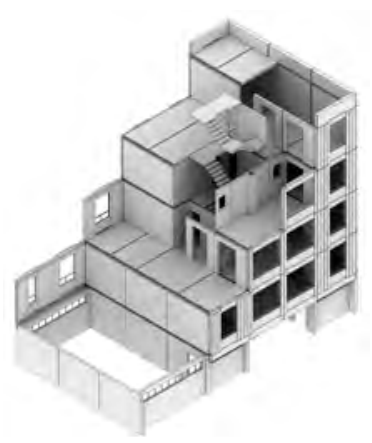

Axonométrica escalonada sistema Larsen \& Nielsen, Dinamarca, años sesenta y setenta. Modelo de Carlos Letelier.

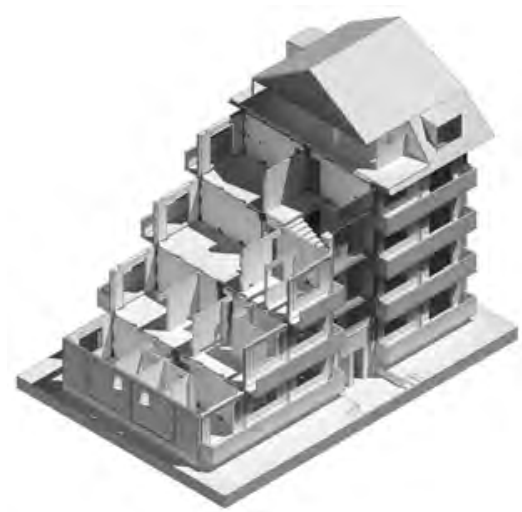

Axonométrica escalonada sistema VEP, Chile, años setenta.

Modelo de Alan Patrick.

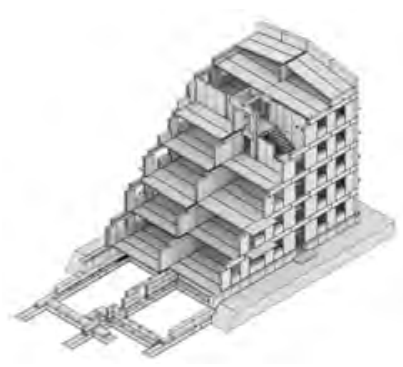

Axonométrica escalonada sistema 1-510, URSS, años cincuenta.

Modelo de Davor Pantoja.

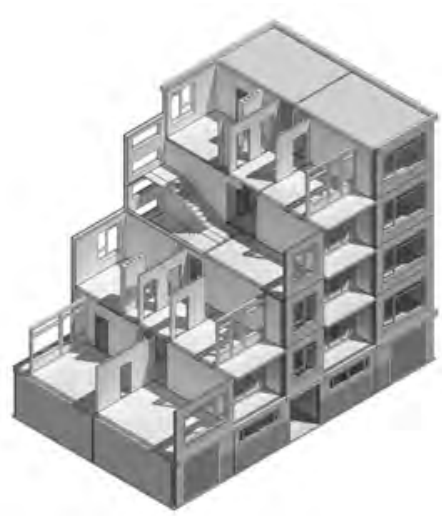

Axonométrica escalonada sistema Coignet, Francia, años cincuenta y sesenta. Modelo de Catalina de Pablo.

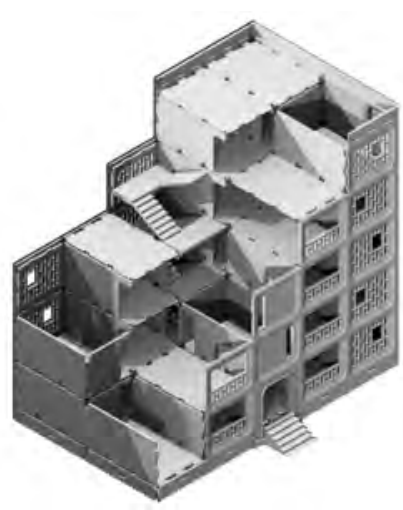

Axonométrica escalonada sistema Gran Panel Soviético, Cuba, años sesenta. Modelo de Javier Rosende. 

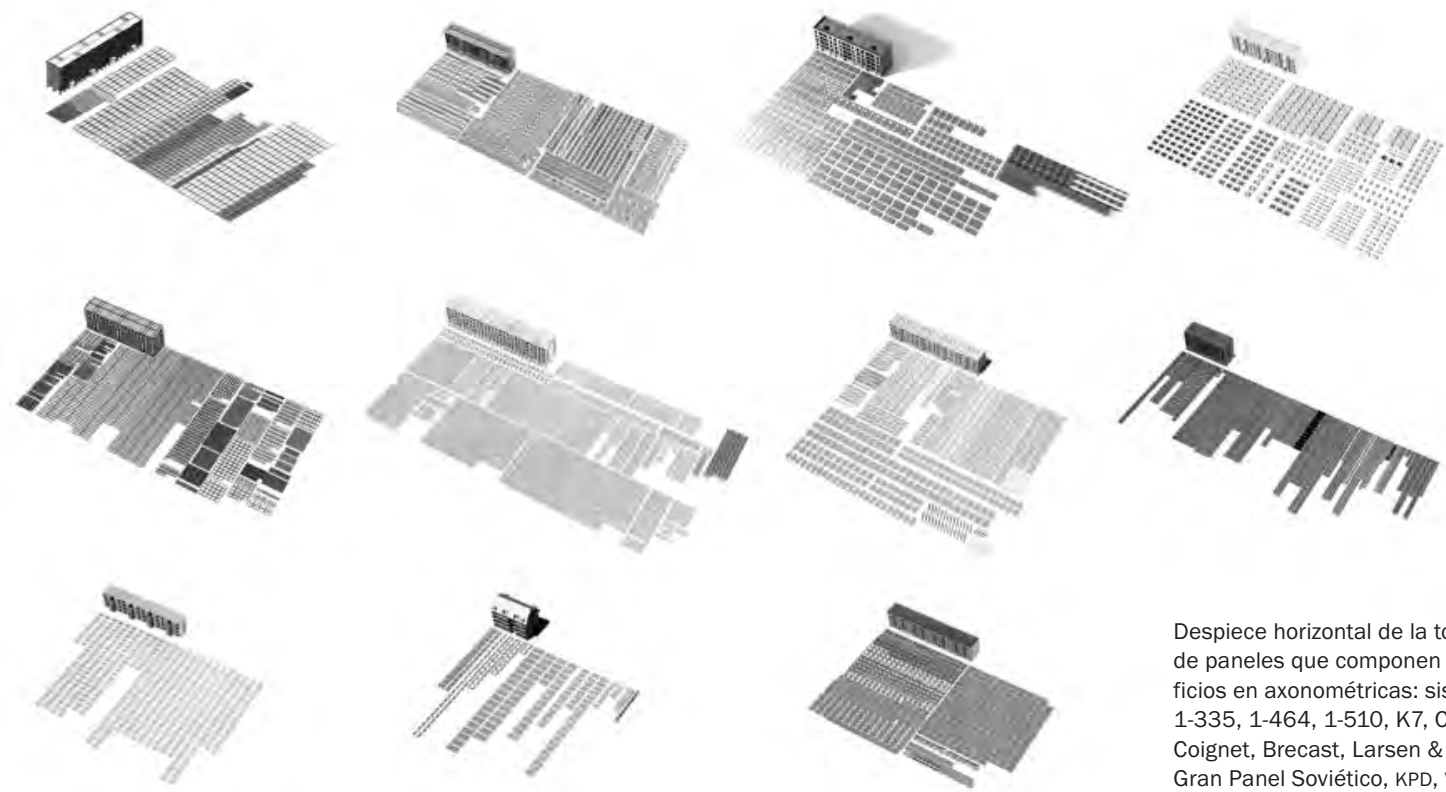

Despiece horizontal de la totalidad de paneles que componen los edificios en axonométricas: sistemas 1-335, 1-464, 1-510, K7, Camus, Coignet, Brecast, Larsen \& Nielsen, Gran Panel Soviético, KPD, VEP.

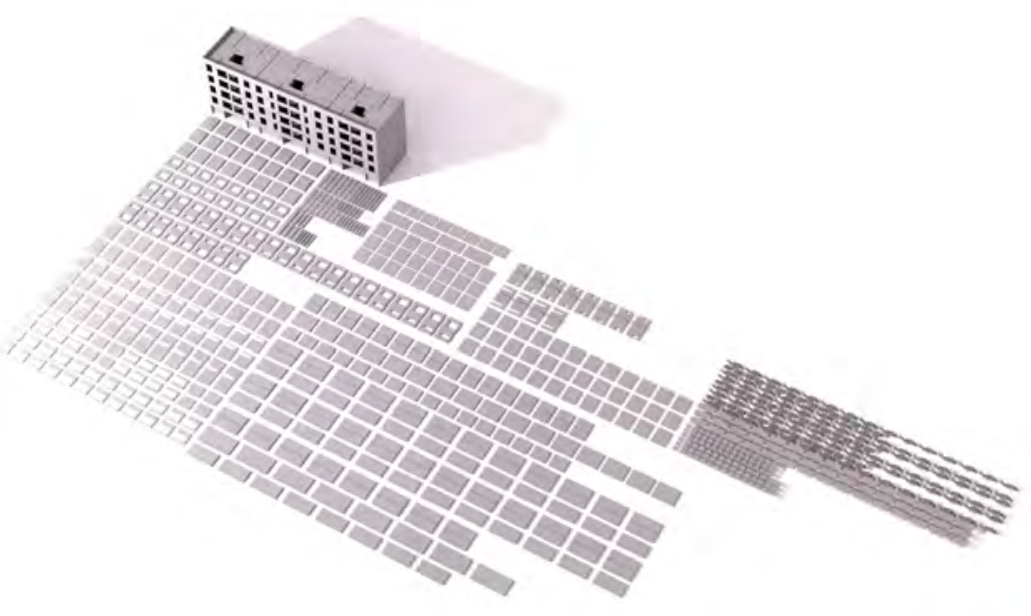

Sistema Larsen \& Nielsen, Dinamarca, años sesenta y setenta. Despiece horizontal de paneles, 647 componentes en total.

Modelo de Carlos Letelier.

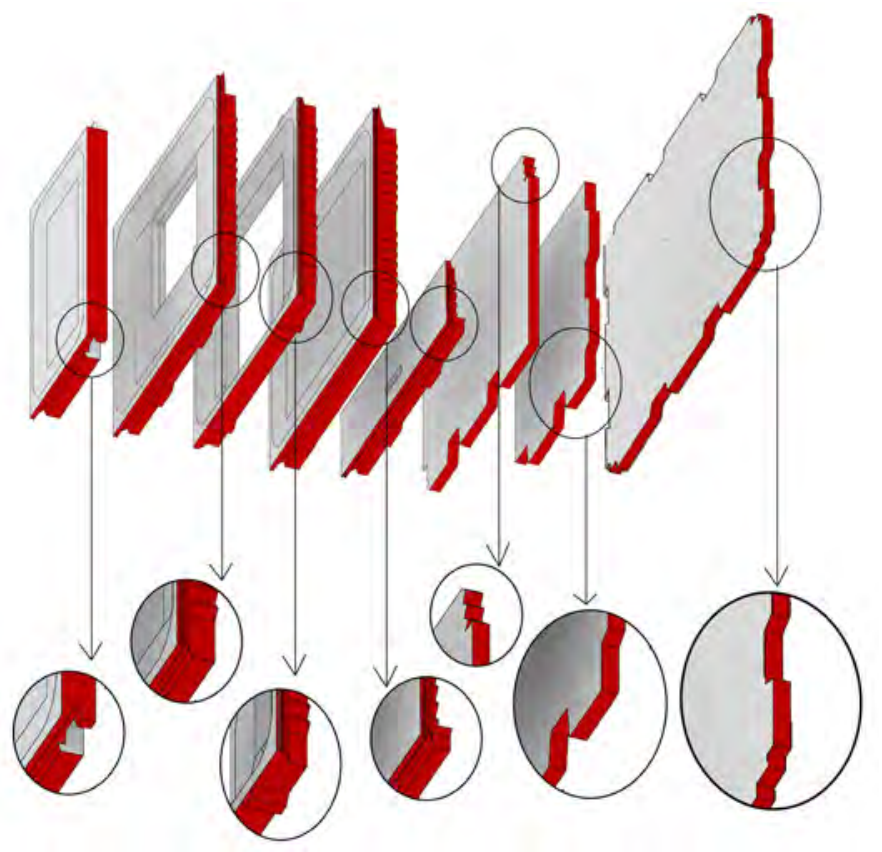

Grilla estructural secundaria sistema KPD, Chile, años setenta. Modelo de Carolina Rocco.

Maquetas escalonadas E. 1: 75, realizadas en impresora de polvo Z310 plus. De izquierda a derecha: sistema Gran Pane Soviético, Cuba, años setenta, modelo de Javier Rosende; Sistema Brecast, Inglaterra, modelo de Constanza Krauss; sistema 1-464, URSS, modelo de Carlos Rojas.

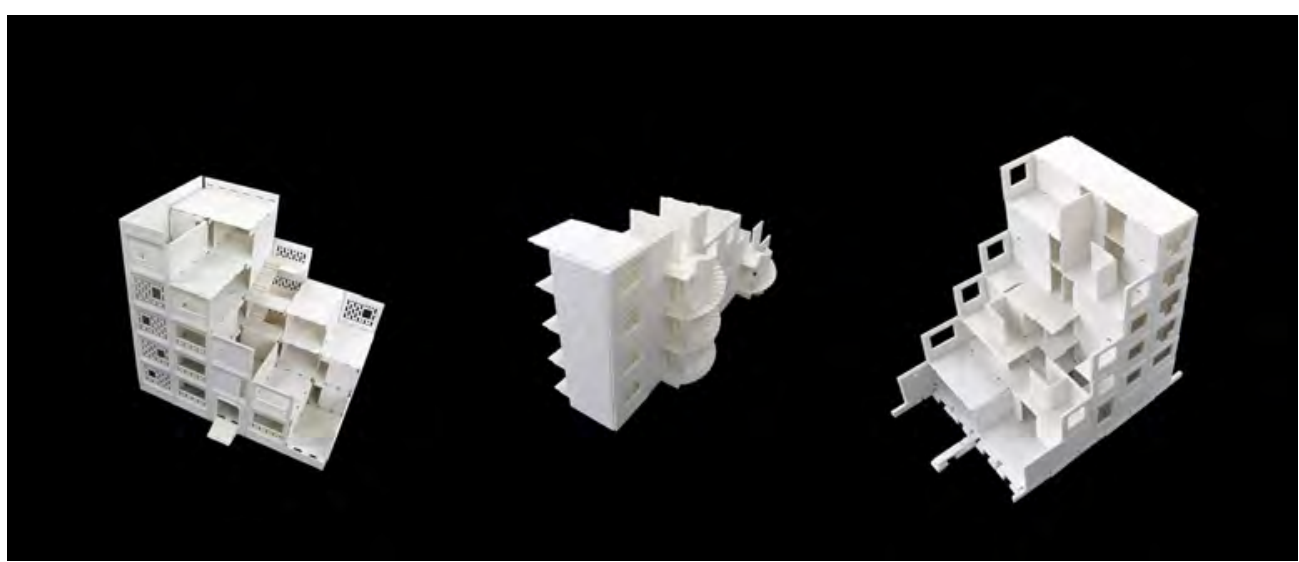




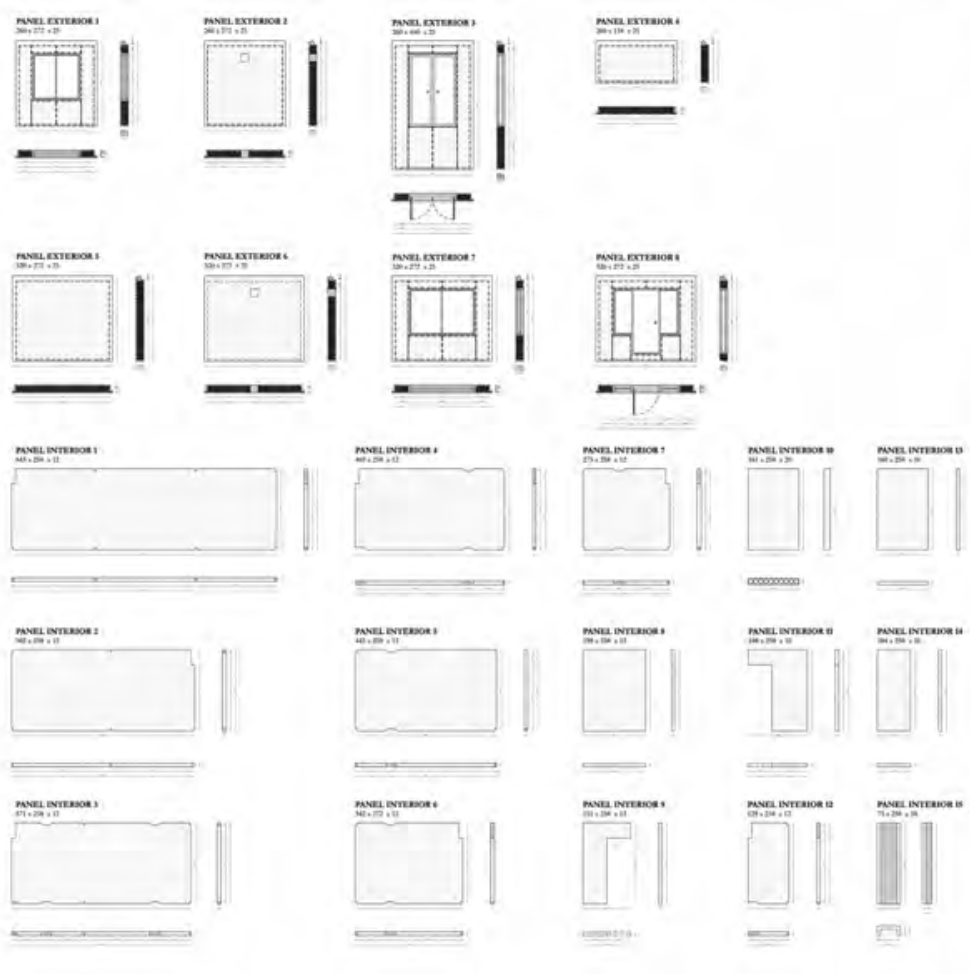

Catálogo razonado de paneles sistema 1-464.

URSS, años cincuenta.

Modelo de Carlos Rojas.

un proceso de diferenciación y selección sujeto a distintas presiones ambientales, técnicas, políticas, estéticas y culturales. El posterior establecimiento de una genealogía a estas series no estaría exento de consideraciones históricas. Por el contrario, esta rechazaría el uso meta-histórico de significaciones ideales y teleologías indefinidas al oponerse de manera consiente a una búsqueda por sus “orígenes" (Foucault, 1971).

Planteado de este modo, no se trataba de imprimir una maqueta para obtener la forma final de un edificio desde un legajo de planos bien conocido. Interesaba, por el contrario, utilizar la reconstrucción del sistema como excusa para identificar los problemas que ingenieros y arquitectos enfrentaron en el desarrollo de las soluciones técnicas y tipológicas de estas series. Esto significó invertir el estatus del objeto de estudio en la relación que en cada caso se establecía entre información disponible y faltante.

Este proceso recordó el comentario de Slavoj Žižek sobre una cita atribuida a Donald Rumsfeld, Secretario de Defensa de los Estados Unidos (2001 y 2006) durante la durante la invasión norteamericana a Irak y Afganistán. Rumsfeld habría dicho: "Hay known knowns; que son cosas que sabemos que sabemos. Hay también known unknows, vale decir cosas que sabemos que no sabemos. Pero también hay unknown unknowns, que son cosas que no sabemos que no sabemos" (Žižek, 2004). Al recordar a Rumsfeld, Žižek tenía un interés psicoanalítico, pues propuso agregar a la lista los unknown knowns, vale decir, aquellas cosas que no sabemos que sabemos. En el contexto de la investigación sobre sistemas constructivos, la connotación "estratégico-militar" de este episodio supuso identificar con claridad lo que no se sabe, para así llenar los vacíos de información a partir de los unknown knowns de Žižek, aquellas
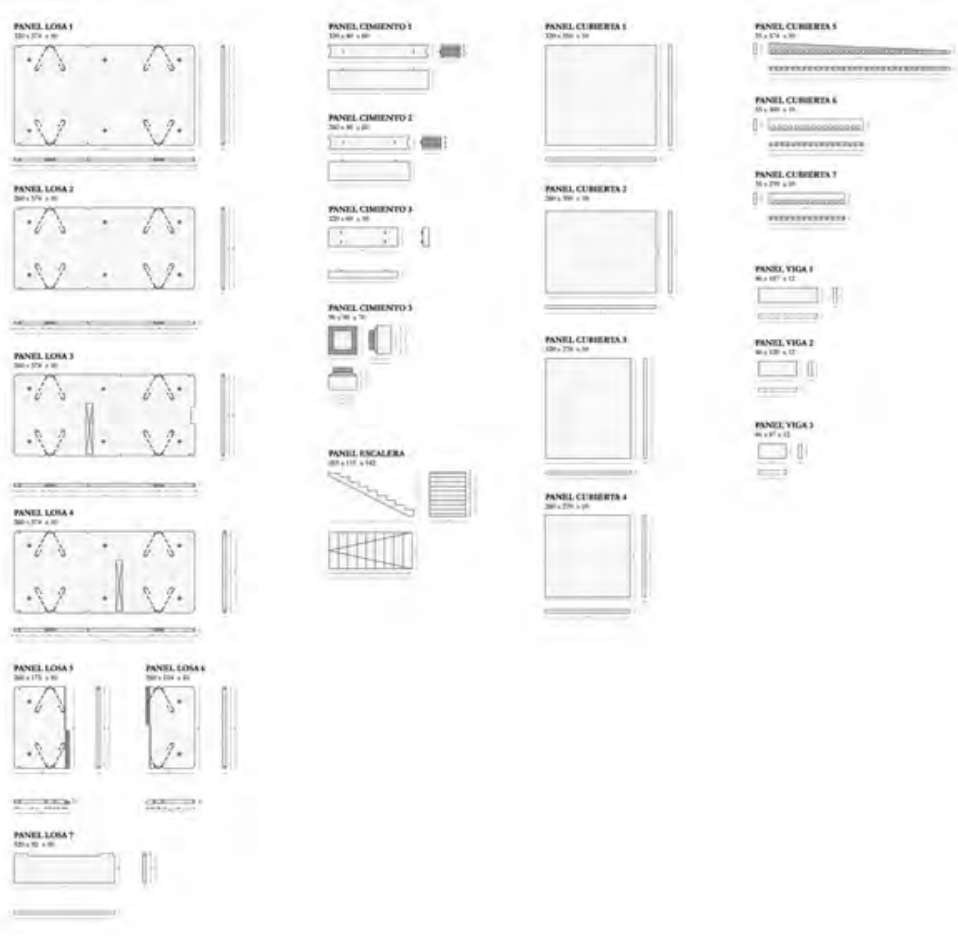

claves de proyecto pertenecientes a una cultura arquitectónica y tecnológica que muchas veces no sabemos que sabemos. El modelo final del sistema, en este sentido, debía ser fiel al proceso argumentativo propio a los materiales presentes y también ausentes.

El caso de algunos sistemas, sin embargo, supuso el problema inverso. Vale decir, la existencia de un exceso de información que obligó a reemplazar la imagen del paleontólogo por la del editor. KРD у Camus, por ejemplo, exigieron a ser muy selectivos en el tipo de documentación considerada relevante. Común a ambas estrategias fue la necesaria vinculación los sistemas a edificios específicos, sobre todo en aquellos casos que permitían gran variedad tipológica a partir de variaciones del propio sistema. David Icekson en su investigación sobre Camus actuó literalmente como arquitecto, diseñando un edificio a partir de antecedentes que permitían una enorme cantidad tipologías posibles.

La relevancia de estudiar estos sistemas en términos comparativos permitió observar las distintas lógicas operando en cada uno de ellos. Precisamente el descubrimiento de problemas específicos por sistema fue el objetivo último del ejercicio de reconstrucción. De origen ruso y llegado Chile en su versión cubana de la década de los sesenta, el sistema K PD permitió a Carolina Rocco identificar una grilla estructural "invisible”, definida por la continuidad vertical y horizontal de una filigrana de hormigón armado conceptualmente auto-portante. En relación con esta, los paneles estructurales emergieron en una dimensión de encofrado para

3 En el caso de los sistemas Taisei (Japón), E-101 (URSS) y Descon/Concordia (EE.UU./ Canadá), se obtuvo información de sus creadores, Hiroshi Yoshida, Leonid Ghelphand y Milo Shemie, respectivamente. 

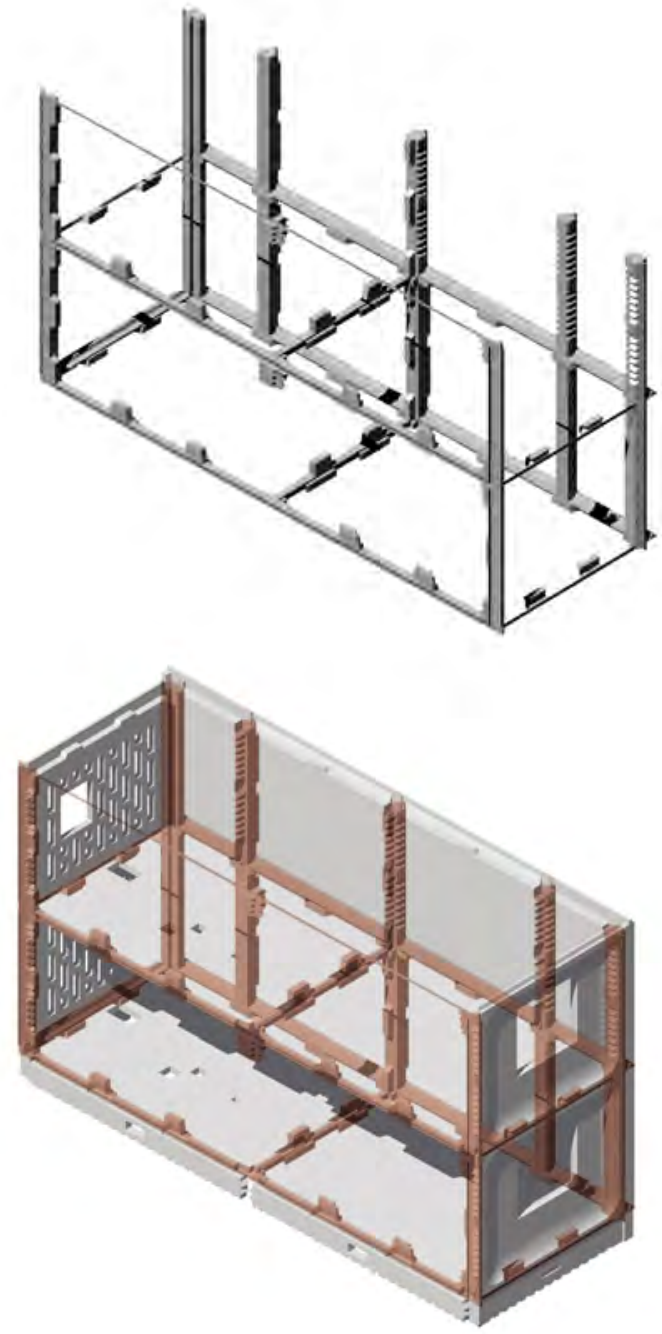

Diagrama grilla estructural y ensamblaje sistema KPD, Chile, años setenta. Modelo de Carolina Rocco. una estructura colaborante, revelando así la importancia de la definición variable de perímetro dentado de los mismos paneles. En Cuba, este sistema había ya sido adaptado a las condiciones climáticas locales a través del aligeramiento y perforación de sus paneles de fachada. En la investigación de Javier Rosende, la adaptación del Gran Panel Soviético (GPs) tuvo el objetivo adicional de lograr variabilidad en la fachada por medio de nueve paneles distintos y sus combinaciones.

El análisis de las características especificas de cada sistema y estudio comparado entre ellos se tradujo en cada caso en el establecimiento de la relación entre el número de tipos de panel y el número total de paneles en el edificio. Es así que mientras el sistema 1-510 requería 57 tipos de panel y 2.499 componentes en total (57/2.499), sucesivamente: 1-335 (39/1.444); Camus (85/1.250); K7 (25/1014); 1-464 (45/994); Coignet (58/946); KрD (35/828); GPS (27/720); Taisei (32/678); Larsen \& Nielsen (44/647); Brecast $(20 / 498)$ y Vep $(17 / 175)$. Esta relación cambiante permitió especular sobre la naturaleza del diseño de cada sistema en relación con la forma final del edificio, pero sobre todo en el tipo de procesos industriales que los hicieron posible. Adrian Forty nos recuerda que fue precisamente la sistematización industrial del panel prefabricado, a través del su "gestión científica" (scientific management) la que vino a transformar este material "arcaico" en uno definitivamente moderno. No por casualidad, dice Forty, Frederick Taylor publicó un Tratado sobre el concreto (Treatise on Concrete, 1905) seis años antes que su obra más conocida e influyente, Los principios de la gestión científica (Principles of Scientific Management) (Forty, 2012). Todos estos sistemas -desarrollados y construidos entre 1948 y 1978- fueron concebidos desde el concreto hacia la abstracción del panel como componente último de la composición plástica.

La impresión digital del modelo permitió revisar la exactitud del material documental disponible y, en muchos casos, detectar equívocos e inconsistencias en las fuentes originales. El uso de herramientas digitales en este tipo de investigación supuso, en efecto, que no se trataba de redibujar edificios y sus tipologías sino de ensamblar componente por componente al interior del modelo. Así, la maqueta adquirió un estatus específico alejado de la mera representación de un edificio, transformándose en herramienta para la corroboración de la exactitud y pertinencia de tesis proyectuales basadas en "suposiciones informadas". ARQ

Pedro Alonso | Arquitecto y Magíster en Arquitectura, Pontificia Universidad Católica de Chile, 2000 y Doctor en Arquitectura (Ph.D), The Architectural Association, 2008. Desde 2005 enseña en The Architectural Association, donde actualmente es profesor visitante en el programa de Master in History and Critical Thinking. En 2010 obtuvo una beca de investigación de The Getty Research Institute y en 2011 fue académico visitante en The Canadian Centre for Architecture CCA - Montreal. Actualmente es profesor de la Escuela de Arquitectura y jefe del programa de Magíster en Arquitectura MARQ de la Pontificia Universidad Católica de Chile.

Hugo Palmarola | Diseñador, Pontificia Universidad Católica de Chile, 2004 y Magíster en Teoría e Historia del Diseño Industrial por Universidad Nacional Autónoma de México, 2010. Fue profesor e investigador en la Pontificia Universidad Católica de Chile. En 2007 recibió The International Scholar Award de SHOT y en 2008, junto a Pedro Alonso, recibió The RIBA Research Trust Award. En 2012, a propósito de sus estudios sobre prefabricación, fue invitado como investigador por Indiana University y por la Universidad de Oriente en Santiago de Cuba.

Bibliografía

BEER, Gillian (ed.). "Introduction”. En DARWIN, Charles. On the Origin of Species. Original de 1859. Oxford University Press, Nueva York, 2008.

CUVIER, Georges. Recherches sur les Ossements Fossiles. Discours préliminaire. Vol. 1. Original de 1812. Traducción de Robert KERR. Essay on the Theory of the Earth. Original de 1813. Gregg Publishing, Nueva York, 1971.

DARWIN, Charles. On the Origin of Species. Original de 1859. Oxford University Press, Nueva York, 2008, p. 229.

FLYNT, Henry y Georges MACIUNAS. Communist must give revolutionary leadership in culture. World View Publishers, Nueva York, 1965

FORTY, Adrian. Concrete and Culture: a Material History. Reaktion Books, Londres, 2012, p. 236. RABINOW, Paul (ed.). The Foucault Reader. Pantheon Books, Nueva York, 1984 ŽIŽEK, Slavoj. "What Rumsfeld Doesn't Know That He Knows About Abu Ghraib". En In These Times. http://www.inthesetimes.com/article/747/, 21 de mayo de 2004. 